\title{
Humanitarian Assistance for Wild Animals
}

\section{Kyle Fohannsen argues that most wild animals live bad lives, and we should intervene in nature to improve their wellbeing}

When you read the title of this paper, what likely comes to mind is images of Koala bears being rescued from bush fires, or of injured raccoons and deer, being rehabilitated after a hurricane. These are examples of humanitarian assistance for wild animals, but they're not what this paper is primarily about. The need for humanitarian assistance in the wild far exceeds the damage caused by natural disasters. Severe suffering is pervasive in nature. It's built into natural processes, and thus it's the norm rather than the exception.

\section{Severe suffering is pervasive in nature}

Many people think that evolution is an inherently beneficial process: that the traits it produces evolved because they benefit the animals who have them. This is a misconception, though. Evolution selects traits that protect an animal's genes, and protecting an animal's genes is different from benefitting her. Richard Dawkins provides some good examples in his book River Out of Eden: male peacocks are encumbered by heavy feathers; and male songbirds exert considerable en- ergy, and attract the attention of predators, by singing for prolonged periods of time. Being encumbered isn't good for peacocks, and exposing themselves to danger isn't good for songbirds. Rather, the function of heavy feathers and prolonged singing, is to protect the birds' genes by attracting mates. Mating, and reproducing, may be enjoyable for these animals, but surely evolution would have facilitated these goals some other way if the purpose of evolved traits were to benefit the animals who have them.

Since the purpose of evolution is to protect genes, you'd think that a parent's evolved traits at least function to benefit her children. Unfortunately, protecting an animals' genes doesn't always benefit her children either. After all, many animals are r-strategists: they protect their genes by producing large numbers of offspring. Some r-strategist parents (such as certain rodents) produce dozens of offspring per litter or clutch, while others (such as certain fish) produce thousands or even millions. Unlike K-strategists, who have few offspring but devote a lot of energy to each of them, r-strategists play a numbers game. Though the majority of their offspring die painfully and prematurely, parents produce enough to ensure that some will survive to 
maturity and successfully reproduce. In other words, the r-strategy protects a parent's genes, but it sacrifices most of her offspring in the process.

It's worth pausing to think about the r-strategy's implications for wild animals' quality of life. First, it's clear that most r-strategists live bad lives. Their lives are typically short, and they typically die from painful causes such as predation, starvation, exposure, or dehydration. What's more, the fact that infant r-strategists are at a high risk of dying from painful causes suggests that their short lives are characterised by hardships, e.g., by inadequate access to food, by the threat of predators, etc. Only a lucky few eventually learn to manage the dangers of life and become comfortable in their environment. Were the r-strategy very rare among wild animal species, its implications might not be too dramatic. Unfortunately, it's prevalent among many species of animal, such as fish, lizards, amphibians, and small mammals. There are many K-strategist species too, of course, but the fact that r-strategists have far higher birth rates than $\mathrm{K}$-strategists, means that far more individual r-strategists are born into the world than individual K-strategists. Given how numerous r-strategists are, ethicists such as Catia Faria, Oscar Horta, Eze Paez, and Brian Tomasik conclude that the amount of suffering in nature probably exceeds the amount of enjoyment in it. They may or may not be right about that, but at the very least we can say that most individual wild animals live bad lives.

It might be objected that most r-strategists aren't sentient: that they're incapable of having experiences such as pleasure or pain. Indeed, many r-strategists are insects, and it's uncertain whether insects are sentient. However, it's important to keep in mind that the r-strategy isn't just common among insects. It's common across many species of animal, including animals we know are sentient, such as mammals (small mammals are often r-strategists). Though it may turn out to be false that most r-strategists are sentient, it remains true that most sentient individuals born into the world are r-strategists. This follows from the fact that sentient r-strategists' birth rates are so much higher than sentient K-strategists'.

Though animals who survive to maturity normally live better lives than those who don't, it would be hasty to conclude that their lives are easy. Mature wild animals are subject to hardships far worse than anything most human beings deal with (especially humans living in wealthy countries). Examples include disease, parasites, predator-induced stress, predator-caused injuries, and extreme temperatures.

\section{Most individual wild animals live bad lives}

Since wild animals are so numerous, and live such difficult lives, the scale of wild animal suffering is far higher than anything we're used to. Consider two well-known, large-scale problems: global poverty and factory farming. According to The World Bank, 689 million human beings were living on less than $\$ 1.90$ per day in 2017. And according to a recent estimate in Christopher Schlottmann's and Jeff Sebo's Food, Animals, and the Environment, 70 billion terrestrial animals are killed every year 
(mostly in factory farms). As large as these numbers are, though, wild animals are far more numerous. For example, the number of sentient $r$-strategist infants who perish over, say, a 5-year period, may very well be somewhere in the quadrillions (or higher).

Despite its scale, wild animal suffering is also neglected. Relative to global poverty and factory farming, very few people are working on ways to assist wild animals. As a result, wild animal suffering presents a tremendous opportunity for philanthropy. Effective interventions would do a tremendous amount of good, and the fact that relatively few people are working on the problem suggests that many potential interventions are still waiting to be discovered. Thankfully, some organisations are currently working on wild animal suffering, e.g., Animal Ethics, Wild Animal Initiative, and Rethink Priorities.

You may be surprised to learn that some beneficial interventions are already being performed on a regular basis. It's standard in many countries to vaccinate wild animal populations against diseases that threaten either domesticated animals or human beings, e.g., rabies. These vaccinations aren't provided for wild animals' sake, but they do foreseeably benefit the wild animals who receive them. One straightforward possibility, then, is to just increase the number of wild animal vaccination programs, and to vaccinate wild animals against diseases even when those diseases don't threaten us.

Restricting ourselves to familiar, relatively small-scale (but still important) interventions isn't very satisfying, though. Perhaps we should go further? We could, for example, research genetic interventions intended to change carnivores into herbi- vores, or r-strategists into K-strategists. Though I think ecosystems populated by K-strategist herbivores would be far better for wild animals than our current ecosystems are, we should only bring such ecosystems about if, and when, it's feasible and safe to do so. Furthermore, it may turn out that it often isn't, and never could be, feasible or safe to bring them about. Put another way, ecosystems populated by K-strategist herbivores are ideal for animals, but at the present moment, it's hard to say whether that ideal could one day be achieved.

\section{Very few people are working on ways to assist wild animals}

One promising type of large-scale intervention, is to give wild animals a "genetic painkiller". In other words, we could use gene-editing to reduce the extent to which animals' pain bothers them (much the way we use medication to dull our own pain). This might sound like science fiction, but it isn't. Gene-editing has already been used to produce mice who have a dulled capacity for pain, and the Sculpting Evolution group at the MIT Media Lab is currently working to produce more. What's more, current gene-editing technology is capable of driving traits through populations, i.e., it can be used to ensure that when an edited animal mates with a wild animal, nearly all of the edited animal's offspring will inherit the engineered trait, that nearly all of her offspring's offspring will inherit the engineered trait, etc. Though it would be foolish to com- 
pletely deprive animals of their capacity for pain, a genetic painkiller wouldn't do that. It would just reduce the extent to which an animal's pain bothers her, thus leaving intact her ability to avoid harmful stimuli. Nor would it be necessary to permanently dull animals' capacity for pain. We could presumably limit the period of time during which an animal's capacity is affected, e.g., we could relieve $r$-strategist infants' pain during the period of time they're most likely to die a painful death, after which their full capacity for pain would return (much the way pain-killing medication works). Interventions like this shouldn't take long to develop, since we've already managed to create a genetic painkiller for mice, and implementing them shouldn't have a significant impact on animals' population sizes or on the way ecosystems function. What they would significantly impact, though, is wild animals' wellbeing. Since the benefits promise to be astronomically large, and the harms quite small, the main challenge is mustering the political will needed to develop, and administer, genetic painkillers to wild animals.

It might be objected that intervening in nature reduces its naturalness, and that naturalness ought to be preserved. However, I don't think it's plausible to claim that naturalness has any value independent of animals', and humans', wellbeing. When interfering with nature improves our own wellbeing, we don't hesitate to do it, e.g., we protect ourselves from bad weather, cure ourselves of diseases, etc. And though intervening in nature carries certain risks, the right response is to proceed with caution. If we have good reason to believe that an intervention will provide significant bene- fits, and good reason to believe that it's safe, then we have good reason to implement it.

A second objection is that intervening in nature is too paternalistic, i.e., that trying to help wild animals requires unjustified, coercive interference with their lives. I have a couple of points to make in reply. First, many interventions do not interfere with wild animals' liberties. For example, using feeding stations to vaccinate wild animals does not involve any coercion at all. Since paternalistic interventions are, by definition, coercive, interventions that don't use coercion can't be paternalistic.

\section{So, what should we do right now?}

Gene-editing, by contrast, does involve some coercion: it requires infringing the liberties of animals who are used in experiments. However, the animals who stand to benefit from these experiments do not have to be coerced in any way. Engineered traits are passed on to future animals by releasing edited animals into the wild. Neither the wild animals who mate with them, nor the generations of offspring that follow, are coerced. However, even if it was true that such interventions are, in some sense, paternalistic, it isn't clear that this is a problem. Paternalistic interference is only inappropriate when a being is competent to manage the dangers she's being protected from. Since most wild animals die during infancy from the various dangers they're exposed to, it's false that they competently manage those dangers. Though it's true that certain wild animal species are competent (those that 
manage to stave off extinction), the vast majority of wild animal individuals are not, and never become, competent.

A third objection is that some interventions, namely those involving gene-editing, require that we first perform unjustified experiments on animals. Genetic experiments require that we restrict animals' liberties, they're often painful, and they can have unintended consequences that negatively impact edited animals' health. Though these are significant harms, a few things about them should be kept in mind. First, some interventions shouldn't require much testing to develop. As I mentioned above, we've already managed to create a genetic painkiller for mice, so we won't need to start from scratch when developing similar painkillers for use in the wild. Second, the harms of genetic experimentation, though significant, only affect a small portion of the targeted populations. As I mentioned above, engineered traits are driven through populations when edited animals reproduce with wild animals. The generations of offspring who inherit the engineered traits would never see the inside of a laboratory, and they would vastly outnumber the animals experimented upon. Though we should be reluctant to benefit some when we must harm others in the process, we should also make exceptions when the stakes are high enough. Since the benefits of administering genetic painkillers to $r$-strategists would be astronomically high, the harms of experimentation are justified. That said, it might be best to wait until there's sufficient public support. It would be wrong to perform experiments unless we have good reason to believe that the interventions under development will actually be implemented.
So, what should we do right now? What are the implications of wild animal suffering for animal activism? Though it might be tempting to advocate for genetic editing, I think it's best to hold off for now. Philosophers and animal studies scholars are sometimes willing to entertain the idea that we should genetically edit wild animal populations, but I don't think the general public will take such proposals seriously (yet). A better strategy is to focus on raising awareness about the full extent of wild animal suffering, and on investigating and building support for comparatively modest interventions, such as wild animal vaccination programs. Once there's sufficient awareness about wild animal suffering, and sufficient support for a range of modest interventions, building support for more ambitious initiatives will be easier. In addition, traditional animal activism has a helpful role to play, e.g., advocating for veganism and for the view that animals matter just as much as we do. People who see animals as our moral equals, and who've gone vegan out of respect for animals' moral importance, are far more likely to care about wild animal suffering when made aware of it. Moral progress can be slow, so there's no time like the present to start.

Kyle Fohannsen is adjunct assistant professor at Queen's University and author of Wild Animal Ethics: The Moral and Political Problem of Wild Animal Suffering (New York: Routledge, 2021). He's does work in political philosophy, and in animal and environmental ethics. 